\title{
Stability and periodicity for impulsive neural networks with delays
}

Yuanfu Shao ${ }^{* *}$ and Qianhong Zhang ${ }^{2}$

"Correspondence:

shaoyuanfu@163.com

${ }^{1}$ College of Science, Guilin

University of Technology, Guilin

Guangxi, 541004, P.R. China

Full list of author information is

available at the end of the article

\begin{abstract}
By constructing a suitable Lyapunov functional and using some inequalities, we investigate the existence, uniqueness and global exponential stability of periodic solutions for a class of generalized cellular neural networks with impulses and time-varying delays. Finally, an illustrative example and simulations are given to show the effectiveness of the main results.
\end{abstract}

Keywords: periodicity; stability; impulsive; delays

\section{Introduction}

The dynamics of cellular neural networks has been deeply investigated due to its applicability in solving image processing, signal processing and pattern recognition problems $[1$, 2]. Recently, the study of the existence and exponential stability of periodic solutions for neural networks has received much attention and many known results have been obtained [3-27]. For example, authors [11] considered the following neural networks with delays:

$$
\begin{aligned}
\dot{x}_{i}(t)= & -a_{i}(t) x_{i}(t)+\sum_{j=1}^{n} b_{i j}(t) f_{j}\left(x_{j}(t)\right)+\sum_{j=1}^{n} c_{i j}(t) f_{j}\left(x_{j}\left(t-\tau_{i j}(t)\right)\right) \\
& +\sum_{j=1}^{n} \sum_{l=1}^{n} d_{i j l}(t) f_{j}\left(x_{j}(t)\right) f_{l}\left(x_{l}(t)\right)+\sum_{j=1}^{n} \sum_{l=1}^{n} e_{i j l}(t) f_{j}\left(t-\sigma_{i j l}(t)\right) \\
& \times f_{l}\left(x_{l}\left(t-\sigma_{i j l}(t)\right)\right)+I_{i}(t),
\end{aligned}
$$

when $\tau_{i j}(t)=\tau_{i j}, \sigma_{i j l}(t)=\sigma_{i j l}$ are constants, a set of easily verifiable sufficient conditions guaranteeing the existence and globally exponential stability of one periodic solution was derived.

However, in practice, impulsive effects are inevitably encountered in implementation of networks, which can also be found in information science, electronics, automatic control systems and so on (see $[12,13,17-19,21,22,24-27])$. Thus it is necessary to study the impulsive case of system (1).

Motivated by the discussion, in this paper, by employing some inequalities and constructing a suitable Lyapunov functional, we aim to investigate the existence and exponential stability of a periodic solution for a class of nonautonomous neural networks with

\section{Springer}

(c)2013 Shao and Zhang; licensee Springer. This is an Open Access article distributed under the terms of the Creative Commons Attribution License (http://creativecommons.org/licenses/by/2.0), which permits unrestricted use, distribution, and reproduction in any medium, provided the original work is properly cited. 
impulses and time-varying delays as follows:

$$
\begin{aligned}
\dot{x}_{i}(t)= & -a_{i}(t) x_{i}(t)+\sum_{j=1}^{n} b_{i j}(t) f_{j}\left(x_{j}(t)\right)+\sum_{j=1}^{n} c_{i j}(t) f_{j}\left(x_{j}\left(t-\tau_{i j}(t)\right)\right) \\
& +\sum_{j=1}^{n} \sum_{l=1}^{n} d_{i j l}(t) f_{j}\left(x_{j}(t)\right) f_{l}\left(x_{l}(t)\right)+\sum_{j=1}^{n} \sum_{l=1}^{n} e_{i j l}(t) f_{j}\left(t-\sigma_{i j l}(t)\right) \\
& \times f_{l}\left(x_{l}\left(t-\sigma_{i j l}(t)\right)\right)+I_{i}(t), \quad t \neq t_{k}, \\
\Delta x_{i}\left(t_{k}\right)= & e_{i k}\left(x_{i}\left(t_{k}^{-}\right)\right), \quad t=t_{k},
\end{aligned}
$$

where $i, j, l=1,2, \ldots, n, n$ corresponds to the number of units in the neural networks. $x_{i}(t)$ corresponds to the state variable at time $t, f_{j}$ is the activation of the neurons. $I_{i}(t)$ is the external bias at time $t .0 \leq \tau_{i j}(t) \leq \tau, 0 \leq \sigma_{i j l}(t) \leq \sigma$ corresponds to the transmission delay respectively, $\tau=\max \tau_{i j}^{+}, \tau_{i j}^{+}=\max _{0 \leq t \leq \omega}\left|\tau_{i j}(t)\right|, \sigma=\max \sigma_{i j l}^{+}, \sigma_{i l j}^{+}=\max _{0 \leq t \leq \omega}\left|\sigma_{i j l}(t)\right|$. The fixed moments of time $t_{k}$ satisfy $t_{1}<t_{2}<\cdots, \lim _{k \rightarrow \infty} t_{k}=\infty . \Delta x_{i}\left(t_{k}\right)=x_{i}\left(t_{k}^{+}\right)-x_{i}\left(t_{k}^{-}\right)$, $x_{i}\left(t_{k}\right)=x_{i}\left(t_{k}^{+}\right)$and $x_{i}\left(t_{k}^{-}\right)$exists. $e_{i k}\left(x_{i}\left(t_{k}^{-}\right)\right)$represents impulsive perturbations of the $i$ th unit at $t_{k}, k=1,2, \ldots$.

By appropriately choosing coefficients, system (2) contains many models as its special cases, which were studied in $[6,8,12,24,25,27]$ respectively.

Throughout this paper, for $i, j, l=1,2, \ldots, n, k=1,2, \ldots, a_{i}(t), b_{i j}(t), c_{i j}(t), d_{i j l}(t), e_{i j l}(t)$, $\tau_{i j}(t), \sigma_{i j l}(t), I_{i}(t)$ are all continuous $\omega$-periodic functions and $[0, \omega] \cap\left\{t_{k}\right\}=\left\{t_{1}, t_{2}, \ldots, t_{q}\right\}$. Further, we suppose that:

$\left(\mathrm{H}_{1}\right)$ There exist positive constants $M_{i}$ and $N_{i}$ such that

$$
\left|f_{i}(u)-f_{i}(v)\right| \leq M_{i}|u-v|, \quad\left|f_{i}(u)\right| \leq N_{i} \quad \text { for all } u, v \in R \text {. }
$$

$\left(\mathrm{H}_{2}\right)$ There exists a constant $L_{i k}$ such that $\left|e_{i k}(u)-e_{i k}(v)\right| \leq L_{i k}|u-v|$ for $u, v \in R$.

$\left(\mathrm{H}_{3}\right)$ There exists a positive integer $q$ such that $t_{k+q}=t_{k}+\omega, e_{i(k+q)}(\cdot)=e_{i k}(\cdot)$.

For convenience, we introduce the following notations:

$$
\begin{array}{ll}
a_{i}^{-}=\min _{t \in[0, \omega]} a_{i}(t)>0, \quad b_{i j}^{+}=\max _{0 \leq t \leq \omega}\left|b_{i j}(t)\right|, \quad c_{i j}^{+}=\max _{0 \leq t \leq \omega}\left|c_{i j}(t)\right|, \\
d_{i j l}^{+}=\max _{0 \leq t \leq \omega}\left|d_{i j l}(t)\right|, \quad e_{i j l}^{+}=\max _{0 \leq t \leq \omega}\left|e_{i j l}(t)\right| .
\end{array}
$$

This paper is organized as follows. In Section 2, preliminaries are introduced. In Section 3, by constructing a suitable Lyapunov functional, the criteria ensuring global exponential stability of a periodic solution for system (2) are established. In Section 4, an example and simulations are shown to illustrate the validity of the main results. Finally, we conclude this paper with a brief discussion in Section 5 .

\section{Preliminaries}

Firstly, we introduce some definitions and lemmas. Let $R^{n}$ be the space of $n$-dimensional real column vectors, and $P C=\left\{f:\left[-\tau^{*}, 0\right] \rightarrow R^{n} \mid f(s)\right.$ is continuous for $s \notin\left(-\tau^{*}, 0\right] \cap$ $\left\{t_{k}\right\}, f\left(s^{+}\right)=f(s), f\left(s^{-}\right)$exists for $\left.s \in\left(-\tau^{*}, 0\right] \cap\left\{t_{k}\right\}, k=1,2, \ldots\right\} . P C_{t}=\left\{f:\left[-\tau^{*}, 0\right] \rightarrow R^{n} \mid f(s)\right.$ 
is continuous for $s \notin\left(-\tau^{*}, 0\right] \cap\left\{t_{k}-t\right\}, f\left(s^{+}\right)=f(s), f\left(s^{-}\right)$exists for $s \in\left(-\tau^{*}, 0\right] \cap\left\{t_{k}-t\right\}, k=$ $1,2, \ldots\}$.

A function $x(t)=\left(x_{1}(t), x_{2}(t), \ldots, x_{n}(t)\right)^{T}:\left[-\tau^{*},+\infty\right) \rightarrow R^{n}$ is called a solution of (2) with the initial condition given by

$$
x(s)=\phi(s) \quad \text { for all } s \in\left[-\tau^{*}, 0\right], \phi \in P C,
$$

if $x(t) \in P C\left[[0,+\infty), R^{n}\right]$ satisfies (2) and (3), where $\tau^{*}=\max _{1 \leq i, j, l \leq n}\left\{\max _{t \in[0, \infty)}\left(\left|\tau_{i j}(t)\right|\right.\right.$, $\left.\left.\left|\sigma_{i j l}(t)\right|\right)\right\}$. We denote a solution through $\phi$ by $x(t, \phi)$ or $x_{t}(\phi), x_{t}(s, \phi)=x(t+s, \phi)$ for all $s \in\left[-\tau^{*}, 0\right], t \geq 0$. Obviously, any solution $x(t, \phi)$ of (2) is continuous at $t \neq t_{k}$ and righthand continuous at $t=t_{k}, t \geq 0$. In addition, we define $\|\phi\|=\sup _{-\tau^{*} \leq s \leq 0}\left(\sum_{i=1}^{n}\left|\phi_{i}(s)\right|^{r}\right)^{\frac{1}{r}}$, where $\phi=\left(\phi_{1}, \phi_{2}, \ldots, \phi_{n}\right) \in P C$ and $r \geq 1$ is a constant.

Definition 1 System (2) is said to be globally exponentially stable if for any two solutions $x(t, \phi)$ and $y(t, \psi)$, there exist some constants $r>0$ and $M \geq 1$ such that

$$
|x(t, \phi)-y(t, \psi)| \leq M\|\phi-\psi\| e^{-r t}
$$

for all $t>0$.

Definition 2 Let $f(t): R \rightarrow R$ be a continuous function, then the Dini right derivative of $f(t)$ is defined as

$$
\frac{D^{+} f(t)}{d t}=\limsup _{h \rightarrow 0^{+}} \frac{f(t+h)-f(t)}{h}
$$

From Definition 2, we can easily obtain the following lemma.

Lemma 1 [12] Let $f(t)$ be a continuous function on $R$. If $f(t)$ is differentiable at $t_{0}$, then

$$
D^{+}\left|f\left(t_{0}\right)\right|= \begin{cases}\dot{f}\left(t_{0}\right) & \text { iff }\left(t_{0}\right)>0 \text { or } f\left(t_{0}\right)=0 \text { and } \dot{f}\left(t_{0}\right)>0 \\ -\dot{f}\left(t_{0}\right) & \text { iff }\left(t_{0}\right)<0 \text { or } f\left(t_{0}\right)=0 \text { and } \dot{f}\left(t_{0}\right)<0 \\ 0 & \text { iff }\left(t_{0}\right)=0 \text { and } \dot{f}\left(t_{0}\right)=0 .\end{cases}
$$

Next, we introduce two important inequalities which play a key role in obtaining the main results.

Lemma 2 (Beckenbach and Bellman) [12, 28] For $a \geq 0, b_{k} \geq 0, k=1,2, \ldots, m$, the following inequality holds:

$$
a \prod_{k=1}^{m} b_{k}^{q_{k}} \leq \frac{1}{r} \sum_{k=1}^{m} q_{k} b_{k}^{r}+\frac{1}{r} a^{r}
$$

where $q_{k}>0$ are some constants and $\sum_{k=1}^{m} q_{k}=r-1, r>1$.

Lemma 3 (Halanay inequality) [12, 24] Assume that $p$, $q$ are constants satisfying $p>q>0$. $g(t)$ is a continuous nonnegative function on $\left[t_{0}-\tau, t_{0}\right]$ satisfying $D^{+} g(t) \leq-p g(t)+q\left\|g_{t}\right\|$ 
for all $t \geq t_{0}$, where $\left\|g_{t}\right\|=\sup _{-\tau \leq s \leq 0}|g(t+s)|$. Then, for all $t \geq t_{0}$, we have

$$
g(t) \leq\left\|g_{t_{0}}\right\| e^{-\lambda\left(t-t_{0}\right)}
$$

where $\lambda$ is the unique positive root of the equation $\lambda-p+q e^{\lambda \tau}=0$.

\section{Main results}

In this section, we construct a suitable Lyapunov functional to study the existence and global exponential stability of periodic solutions of system (2).

Theorem 1 Suppose that $\left(\mathrm{H}_{1}\right)-\left(\mathrm{H}_{3}\right)$ hold. Further,

$\left(\mathrm{H}_{4}\right)$ There exist $r>1, q_{k}>0, \lambda_{i}>0, \alpha_{k i}, \beta_{k i}, \gamma_{k i}$ and $\eta_{k i} \in R(i=1,2, \ldots, n, k=1,2, \ldots, m)$ such that $p>q>0, \sum_{k=1}^{m} q_{k}=r-1$, where

$$
\begin{aligned}
p= & \max _{1 \leq i \leq n}\left[r a_{i}^{-}-\sum_{j=1}^{n} b_{j i}^{+} \frac{\lambda_{j}}{\lambda_{i}} M_{i}^{r\left(1-\sum_{k=1}^{m} \alpha_{k i}\right)}-\sum_{j=1}^{n} b_{i j}^{+} \sum_{k=1}^{m} q_{k} M_{j}^{\frac{r \alpha_{k j}}{q_{k}}}-\sum_{j=1}^{n} c_{i j}^{+} \sum_{k=1}^{m} q_{k} M_{j}^{\frac{r \beta_{k j}}{q_{k}}}\right. \\
& -\sum_{j=1}^{n} \sum_{l=1}^{n}\left(d_{i j l}^{+}+d_{i l j}^{+}\right) N_{l} \sum_{k=1}^{m} q_{k} M_{j}^{\frac{r \gamma}{\gamma_{k j}}}-\sum_{j=1}^{n} \sum_{l=1}^{n}\left(d_{j l}^{+}+d_{j l i}^{+}\right) \frac{\lambda_{j}}{\lambda_{i}} N_{l} M_{i}^{1-\sum_{k=1}^{m} \gamma_{k i}} \\
& \left.-\sum_{j=1}^{n} \sum_{l=1}^{n}\left(e_{i j l}^{+}+e_{i l j}^{+}\right) N_{l} \sum_{k=1}^{m} q_{k} M_{j}^{\frac{r \eta}{q_{k j}}}\right] \\
q= & \max _{1 \leq i \leq n}\left[\sum_{j=1}^{n} c_{j i}^{+} \frac{\lambda_{j}}{\lambda_{i}} M_{i}^{r\left(1-\sum_{k=1}^{m} \beta_{k i}\right)}+\sum_{j=1}^{n} \sum_{l=1}^{n} \frac{\lambda_{j}}{\lambda_{i}}\left(e_{j l}^{+}+e_{j l i}^{+}\right) N_{l} M_{i}^{\left.r\left(1-\sum_{k=1}^{m} \eta_{k i}\right)\right]}\right]
\end{aligned}
$$

$\left(\mathrm{H}_{5}\right)$ There is $0<\mu<\lambda$ such that $\ln \left[\left(1+L_{k}\right) e^{\frac{\lambda \tau}{r}}\right] \leq \frac{\mu\left(t_{k}-t_{k-1}\right)}{r}, k=1,2, \ldots, L_{k}=\max _{1 \leq i \leq n}\left\{L_{i k}\right\}$, $\lambda$ is the unique positive solution of the equation $\lambda-p+q e^{\lambda \tau}=0$.

Then system (2) has a unique $\omega$-periodic solution which is globally exponentially stable.

Proof Let $x(t)=\left(x_{1}(t), x_{2}(t), \ldots, x_{n}(t)\right)^{T}$ and $y(t)=\left(y_{1}(t), y_{2}(t), \ldots, y_{n}(t)\right)^{T}$ be two solutions of (2) through $\phi$ and $\psi$, respectively, where $\phi, \psi \in P C$, then we have

$$
\begin{aligned}
& \frac{d\left(x_{i}(t)-y_{i}(t)\right)}{d t} \\
& =-a_{i}(t)\left(x_{i}(t)-y_{i}(t)\right) \\
& \quad+\sum_{j=1}^{n} b_{i j}(t)\left(f_{j}\left(x_{j}(t)\right)-f_{j}\left(y_{j}(t)\right)\right)+\sum_{j=1}^{n} c_{i j}(t)\left(f_{j}\left(x_{j}\left(t-\tau_{i j}(t)\right)\right)-f_{j}\left(y_{j}\left(t-\tau_{i j}(t)\right)\right)\right) \\
& \quad+\sum_{j=1}^{n} \sum_{l=1}^{n} d_{i j l}(t)\left(f_{j}\left(x_{j}(t)\right) f_{l}\left(x_{l}(t)\right)-f_{j}\left(y_{j}(t)\right) f_{l}\left(y_{l}(t)\right)\right) \\
& \quad+\sum_{j=1}^{n} \sum_{l=1}^{n} e_{i j l}(t)\left(f_{j}\left(x_{j}\left(t-\sigma_{i j l}(t)\right)\right) f_{l}\left(x_{l}\left(t-\sigma_{i l l}(t)\right)\right)\right. \\
& \left.\quad-f_{j}\left(y_{j}\left(t-\sigma_{i j l}(t)\right)\right) f_{l}\left(y_{l}\left(t-\sigma_{i j l}(t)\right)\right)\right) x_{i}\left(t_{k}\right)-y_{i}\left(t_{k}\right) \\
& =x_{i}\left(t_{k}^{-}\right)-y_{i}\left(t_{k}^{-}\right)+e_{i k}\left(x_{i}\left(t_{k}^{-}\right)\right)-e_{i k}\left(y_{i}\left(t_{k}^{-}\right)\right) .
\end{aligned}
$$


Let $z_{i}(t)=x_{i}(t)-y_{i}(t)$. Consider the following Lyapunov functional:

$$
V(t)=\sum_{i=1}^{n} \lambda_{i}\left|x_{i}(t)-y_{i}(t)\right|^{r}=\sum_{i=1}^{n} \lambda_{i}\left|z_{i}(t)\right|^{r}
$$

Calculating the Dini upper right derivative of $V(t)$ along the solution of (2) at a continuous point $t \neq t_{k}$ and applying Lemma 2 , we have

$$
\begin{aligned}
& D^{+}\left(V(t)=\sum_{i=1}^{n} \lambda_{i} D^{+}\left|x_{i}(t)-y_{i}(t)\right|^{r}=\sum_{i=1}^{n} \lambda_{i} r\left|x_{i}(t)-y_{i}(t)\right|^{r-1} D^{+}\left|x_{i}(t)-y_{i}(t)\right|\right. \\
& =\sum_{i=1}^{n} \lambda_{i} r\left|x_{i}(t)-y_{i}(t)\right|^{r-1} \operatorname{sgn}\left(x_{i}(t)-y_{i}(t)\right) \\
& \times\left\{-a_{i}(t)\left(x_{i}(t)-y_{i}(t)\right)+\sum_{j=1}^{n} b_{i j}(t)\left(f_{j}\left(x_{j}(t)\right)-f_{j}\left(y_{j}(t)\right)\right)\right. \\
& +\sum_{j=1}^{n} c_{i j}(t)\left(f_{j}\left(x_{j}\left(t-\tau_{i j}(t)\right)\right)-f_{j}\left(y_{j}\left(t-\tau_{i j}(t)\right)\right)\right) \\
& +\sum_{j=1}^{n} \sum_{l=1}^{n} d_{i j l}(t)\left(f_{j}\left(x_{j}(t)\right) f_{l}\left(x_{l}(t)\right)-f_{j}\left(y_{j}(t)\right) f_{l}\left(y_{l}(t)\right)\right) \\
& +\sum_{j=1}^{n} \sum_{l=1}^{n} e_{i j l}(t)\left(f_{j}\left(x_{j}\left(t-\sigma_{i j l}(t)\right)\right) f_{l}\left(x_{l}\left(t-\sigma_{i j l}(t)\right)\right)\right. \\
& \left.\left.-f_{j}\left(y_{j}\left(t-\sigma_{i j l}(t)\right)\right) f_{l}\left(y_{l}\left(t-\sigma_{i j l}(t)\right)\right)\right)\right\} \\
& \leq \sum_{i=1}^{n} \lambda_{i} r\left\{-a_{i}(t)\left|x_{i}(t)-y_{i}(t)\right|^{r}+\sum_{j=1}^{n} b_{i j}^{+} M_{j}\left|x_{i}(t)-y_{i}(t)\right|^{r-1}\left|x_{j}(t)-y_{j}(t)\right|\right. \\
& +\sum_{j=1}^{n} c_{i j}^{+} M_{j}\left|x_{i}(t)-y_{i}(t)\right|^{r-1}\left|x_{j}\left(t-\tau_{i j}(t)\right)-y_{j}\left(t-\tau_{i j}(t)\right)\right| \\
& +\sum_{j=1}^{n} \sum_{l=1}^{n} e_{i j l}^{+}\left[N_{l} M_{j}\left|x_{i}(t)-y_{i}(t)\right|^{r-1}\left|x_{j}\left(t-\sigma_{i j l}(t)\right)-y_{j}\left(t-\sigma_{i j l}(t)\right)\right|\right. \\
& \left.+N_{j} M_{l}\left|x_{i}(t)-y_{i}(t)\right|^{r-1}\left|x_{l}\left(t-\sigma_{i j l}(t)\right)-y_{l}\left(t-\sigma_{i j l}(t)\right)\right|\right] \\
& +\sum_{j=1}^{n} \sum_{l=1}^{n} d_{i j l}^{+}\left[N_{l} M_{j}\left|x_{i}(t)-y_{i}(t)\right|^{r-1}\left|x_{j}(t)-y_{j}(t)\right|\right. \\
& \left.\left.+N_{j} M_{l}\left|x_{i}(t)-y_{i}(t)\right|^{r-1}\left|x_{l}(t)-y_{l}(t)\right|\right]\right\} \\
& =\sum_{i=1}^{n} \lambda_{i} r\left\{-a_{i}(t)\left|z_{i}(t)\right|^{r}+\sum_{j=1}^{n} b_{i j}^{+} M_{j}\left|z_{i}(t)\right|^{r-1}\left|z_{j}(t)\right|\right. \\
& +\sum_{j=1}^{n} c_{i j}^{+} M_{j}\left|z_{i}(t)\right|^{r-1}\left|z_{j}\left(t-\tau_{i j}(t)\right)\right|
\end{aligned}
$$




$$
\begin{aligned}
& +\sum_{j=1}^{n} \sum_{l=1}^{n} e_{i j l}^{+}\left[N_{l} M_{j}\left|z_{i}(t)\right|^{r-1}\left|z_{j}\left(t-\sigma_{i j l}(t)\right)\right|+N_{j} M_{l}\left|z_{i}(t)\right|^{r-1}\left|z_{l}\left(t-\sigma_{i j l}(t)\right)\right|\right] \\
& \left.+\sum_{j=1}^{n} \sum_{l=1}^{n} d_{i j l}^{+}\left[N_{l} M_{j}\left|z_{i}(t)\right|^{r-1}\left|z_{j}(t)\right|+N_{j} M_{l}\left|z_{i}(t)\right|^{r-1}\left|z_{l}(t)\right|\right]\right\} \\
& =\sum_{i=1}^{n} \lambda_{i} r\left\{-a_{i}(t)\left|z_{i}(t)\right|^{r}+\sum_{j=1}^{n} b_{i j}^{+} M_{j}^{1-\sum_{k=1}^{m} \alpha_{k j}}\left|z_{j}(t)\right| \prod_{k=1}^{m} M_{j}^{\alpha_{k j}}\left|z_{i}(t)\right|^{q_{k}}\right. \\
& +\sum_{j=1}^{n} c_{i j}^{+} M_{j}^{1-\sum_{k=1}^{m} \beta_{k j}}\left|z_{j}\left(t-\tau_{i j}(t)\right)\right| \prod_{k=1}^{m} M_{j}^{\beta_{k j}}\left|z_{i}(t)\right|^{q_{k}} \\
& +\sum_{j=1}^{n} \sum_{l=1}^{n}\left(d_{i j l}^{+}+d_{i l j}^{+}\right) N_{l} M_{j}^{1-\sum_{k=1}^{m} \gamma_{k j}}\left|z_{j}(t)\right| \prod_{k=1}^{m} M_{j}^{\gamma_{k j}}\left|z_{i}(t)\right|^{q_{k}} \\
& \left.+\sum_{j=1}^{n} \sum_{l=1}^{n}\left(e_{i j l}^{+}+e_{i l j}^{+}\right) N_{l} M_{j}^{1-\sum_{k=1}^{m} \eta_{k j}}\left|z_{j}\left(t-\sigma_{i j l}(t)\right)\right| \prod_{k=1}^{m} M_{j}^{\eta_{k j}}\left|z_{i}(t)\right|^{q_{k}}\right\} \\
& \leq \sum_{i=1}^{n} \lambda_{i}\left\{-r a_{i}^{-}\left|z_{i}(t)\right|^{r}+\sum_{j=1}^{n} b_{i j}^{+} M_{j}^{r\left(1-\sum_{k=1}^{m} \alpha_{k j}\right)}\left|z_{j}(t)\right|^{r}+\sum_{j=1}^{n} b_{i j}^{+} \sum_{k=1}^{m} q_{k} M_{j}^{\frac{r \alpha_{k j}}{q_{k}}}\left|z_{i}(t)\right|^{r}\right. \\
& +\sum_{j=1}^{n} c_{i j}^{+} M_{j}^{r\left(1-\sum_{k=1}^{m} \beta_{k j}\right)}\left|z_{j}\left(t-\tau_{j}(t)\right)\right|^{r}+\sum_{j=1}^{n} c_{i j}^{+} \sum_{k=1}^{m} q_{k} M_{j}^{\frac{r \beta_{k j}}{q_{k}}}\left|z_{i}(t)\right|^{r} \\
& +\sum_{j=1}^{n} \sum_{l=1}^{n}\left(d_{i j l}^{+}+d_{i l j}^{+}\right) N_{l}\left[\sum_{k=1}^{m} q_{k} M_{j}^{\frac{r \gamma_{k j}}{q_{k}}}\left|z_{i}(t)\right|^{r}+M_{j}^{r\left(1-\sum_{k=1}^{m} \gamma_{k j}\right)}\left|z_{j}(t)\right|^{r}\right] \\
& \left.+\sum_{j=1}^{n} \sum_{l=1}^{n}\left(e_{i j l}^{+}+e_{i l j}^{+}\right) N_{l}\left[\sum_{k=1}^{m} q_{k} M_{j}^{\frac{r \eta}{q_{k j}}}\left|z_{i}(t)\right|^{r}+M_{j}^{r\left(1-\sum_{k=1}^{m} \eta_{k j}\right)}\left|z_{j}\left(t-\sigma_{i j l}(t)\right)\right|^{r}\right]\right\} \\
& \leq \sum_{i=1}^{n} \lambda_{i}\left[-r a_{i}^{-}+\sum_{j=1}^{n} b_{j i}^{+} \frac{\lambda_{j}}{\lambda_{i}} M_{i}^{r\left(1-\sum_{k=1}^{m} \alpha_{k i}\right)}+\sum_{j=1}^{n} b_{i j}^{+} \sum_{k=1}^{m} q_{k} M_{j}^{\frac{r \alpha_{k j}}{q_{k}}}\right. \\
& +\sum_{j=1}^{n} c_{i j}^{+} \sum_{k=1}^{m} q_{k} M_{j}^{\frac{r \beta_{k j}}{q_{k}}}+\sum_{j=1}^{n} \sum_{l=1}^{n}\left(d_{i j l}^{+}+d_{i l j}^{+}\right) N_{l} \sum_{k=1}^{m} q_{k} M_{j}^{\frac{r \gamma_{k j}}{q_{k}}} \\
& +\sum_{j=1}^{n} \sum_{l=1}^{n}\left(d_{j i l}^{+}+d_{j l i}^{+}\right) \frac{\lambda_{j}}{\lambda_{i}} N_{l} M_{i}^{r\left(1-\sum_{k=1}^{m} \gamma_{k i}\right)} \\
& \left.+\sum_{j=1}^{n} \sum_{l=1}^{n}\left(e_{i j l}^{+}+e_{i l j}^{+}\right) N_{l} \sum_{k=1}^{m} q_{k} M_{j}^{\frac{r \eta_{k j}}{q_{k}}}\right]\left|z_{i}(t)\right|^{r} \\
& +\sum_{i=1}^{n} \lambda_{i}\left[\sum_{j=1}^{n} c_{j i}^{+} \frac{\lambda_{j}}{\lambda_{i}} M_{i}^{r\left(1-\sum_{k=1}^{m} \beta_{k i}\right)}+\sum_{j=1}^{n} \sum_{l=1}^{n} \frac{\lambda_{j}}{\lambda_{i}}\left(e_{j i l}^{+}+e_{j l i}^{+}\right) N_{l} M_{i}^{r\left(1-\sum_{k=1}^{m} \eta_{k i}\right)}\right]\left\|z_{i}\right\|^{r} \\
& \leq-p V(t)+q\left\|V_{t}\right\| .
\end{aligned}
$$

For any $t \in\left[t_{0}, t_{1}\right)$, by $\left(\mathrm{H}_{4}\right)$ and Lemma 3 , we have

$$
|V(t)| \leq\left\|V_{t_{0}}\right\| e^{-\lambda\left(t-t_{0}\right)}
$$


i.e.,

$$
|V(t)|^{1 / r} \leq\left\|V_{t_{0}}\right\|^{1 / r} e^{-\lambda\left(t-t_{0}\right) / r} .
$$

Also, it follows from $\left(\mathrm{H}_{2}\right)$ that

$$
\begin{aligned}
\left|V\left(t_{1}\right)\right|^{1 / r} & \leq\left\{\sum_{i=1}^{n} \lambda_{i}\left(\left|x_{i}\left(t_{1}^{-}\right)-y_{i}\left(t_{1}^{-}\right)\right|+L_{i 1}\left|x_{i}\left(t_{1}^{-}\right)-y_{i}\left(t_{1}^{-}\right)\right|\right)^{r}\right\}^{1 / r} \\
& =\left\{\sum_{i=1}^{n} \lambda_{i}\left(1+L_{i 1}\right)^{r}\left|x_{i}\left(t_{1}^{-}\right)-y_{i}\left(t_{1}^{-}\right)\right|^{r}\right\}^{1 / r} \\
& \leq\left(1+L_{1}\right)\left\|V_{t_{0}}\right\|^{1 / r} e^{-\lambda\left(t_{1}-t_{0}\right) / r} .
\end{aligned}
$$

Therefore, from (6) and (7), for any $t \in\left[t_{0}, t_{1}\right]$, we have

$$
|V(t)|^{1 / r} \leq\left(1+L_{1}\right)\left\|V_{t_{0}}\right\|^{1 / r} e^{-\lambda\left(t-t_{0}\right) / r} .
$$

Similar to (6), for all $t \in\left[t_{1}, t_{2}\right)$, we can derive that

$$
\begin{aligned}
|V(t)|^{1 / r} & \leq\left\|V\left(t_{1}\right)\right\|^{1 / r} e^{-\lambda\left(t-t_{1}\right) / r} \\
& =\left\{\sup _{-\tau^{*} \leq s \leq 0}\left|V\left(t_{1}+s\right)\right|\right\}^{1 / r} e^{-\lambda\left(t-t_{1}\right) / r} \\
& \leq\left(1+L_{1}\right)\left\|V_{t_{0}}\right\|^{1 / r} e^{-\lambda\left(t_{1}-\tau-t_{0}\right) / r} e^{-\lambda\left(t-t_{1}\right) / r}, \\
& \leq\left(1+L_{1}\right) e^{\lambda \tau}\left\|V_{t_{0}}\right\|^{1 / r} e^{-\lambda\left(t-t_{0}\right) / r} .
\end{aligned}
$$

Again by $\left(\mathrm{H}_{2}\right)$, then

$$
\begin{aligned}
\left|V\left(t_{2}\right)\right|^{1 / r} & \leq\left\{\sum_{i=1}^{n} \lambda_{i}\left(\left|x_{i}\left(t_{2}^{-}\right)-y_{i}\left(t_{2}^{-}\right)\right|+L_{i 2}\left|x_{i}\left(t_{2}^{-}\right)-y_{i}\left(t_{2}^{-}\right)\right|\right)^{r}\right\}^{1 / r} \\
& \leq\left(1+L_{1}\right)\left(1+L_{2}\right) e^{\lambda \tau}\left\|V_{t_{0}}\right\|^{1 / r} e^{-\lambda\left(t_{2}-t_{0}\right) / r}
\end{aligned}
$$

Thus, for any $t \in\left(t_{0}, t_{2}\right]$,

$$
|V(t)|^{1 / r} \leq\left(1+L_{1}\right)\left(1+L_{2}\right) e^{\lambda \tau}\left\|V_{t_{0}}\right\|^{1 / r} e^{-\lambda\left(t-t_{0}\right) / r} .
$$

By mathematical induction, for all $t \in\left[t_{k}, t_{k-1}\right)$, we have

$$
|V(t)|^{1 / r} \leq\left(1+L_{1}\right)\left(1+L_{2}\right) \cdots\left(1+L_{k}\right) e^{k \lambda \tau}\left\|V_{t_{0}}\right\|^{1 / r} e^{-\lambda\left(t-t_{0}\right) / r} .
$$

According to $\left(\mathrm{H}_{5}\right),\left(1+L_{k}\right) e^{\lambda \tau / r} \leq e^{\mu\left(t_{k}-t_{k-1}\right) / r}$ for all $k=1,2, \ldots$, hence

$$
\begin{aligned}
|V(t)|^{1 / r} & \leq e^{\mu\left(t_{1}-t_{0}\right) / r} e^{\mu\left(t_{2}-t_{1}\right) / r} \cdots e^{\mu\left(t_{k}-t_{k-1}\right) / r}\left\|V_{t_{0}}\right\|^{1 / r} e^{-\lambda\left(t-t_{0}\right) / r} \\
& \leq\left\|V_{t_{0}}\right\|^{1 / r} e^{-(\lambda-\mu)\left(t-t_{0}\right) / r} .
\end{aligned}
$$


Therefore,

$$
|V(t)|^{1 / r} \leq\left\|V_{t_{0}}\right\|^{1 / r} e^{-(\lambda-\mu)\left(t-t_{0}\right) / r} \quad \text { for all } t \geq t_{0}
$$

i.e.,

$$
|x(t, \phi)-y(t, \psi)| \leq \sqrt[r]{\frac{\lambda_{\max }}{\lambda_{\min }}} e^{-(\lambda-\mu)\left(t-t_{0}\right) / r}\|\phi-\psi\| \quad \text { for all } t \geq t_{0},
$$

where $\lambda_{\max }=\max _{1 \leq i \leq n}\left\{\lambda_{i}\right\}, \lambda_{\min }=\min _{1 \leq i \leq n}\left\{\lambda_{i}\right\}$. This implies that system (2) is globally exponentially stable.

Next, we prove the existence of a periodic solution of (2). Define a Poincáre mapping $T: P C_{0} \rightarrow P C_{\omega}$ as follows:

$$
T(\phi)=x_{\omega}(\phi) \quad \text { for any } \phi \in P C_{0} .
$$

By the periodicity of (2), we can derive that $T^{m}(\phi)=x_{k \omega}(\phi)$ for any integer $m>0$. From the above conclusion, we can choose a positive integer $m$ such that $\sqrt[r]{\frac{\lambda_{\max }}{\lambda_{\min }}} e^{-(\lambda-\mu)(t-m \omega) / r}<1$. According to the periodicity of $\left\{t_{k}\right\}$ and $P C_{0}=P C_{\omega}$, we have

$$
\left\|T^{m} \phi-T^{m} \psi\right\|=\left\|x_{m \omega}(\phi)-x_{m \omega}(\psi)\right\|<\|\phi-\psi\| .
$$

Then operator $T^{m}$ is a construction mapping in $P C_{0}$. Obviously, $P C_{0}$ is a Banach space. By virtue of the Banach fixed point theorem, $T^{m}$ has a unique fixed point $\phi^{*} \in P C_{0}$. On the other hand, $T^{m}\left(T \phi^{*}\right)=T\left(T^{m} \phi^{*}\right)=T\left(\phi^{*}\right)$, then $T \phi^{*}$ is also a fixed point of $T^{m}$. By the uniqueness of fixed point, we obtain that $T \phi^{*}=\phi^{*}$. Therefore, system (2) has a unique $\omega$-periodic solution which is globally exponentially stable. The proof is complete.

If there is no impulse, system (2) reduces to (1) studied in [11]. From the proof of Theorem 1 , we have the following corollary.

Corollary 1 Suppose that $\left(\mathrm{H}_{1}\right)-\left(\mathrm{H}_{4}\right)$ hold. Then system (1) has a unique $\omega$-periodic solution which is globally exponentially stable.

Remark 1 In [11], by using the Mawhin continuation theorem and constructing Lyapunov functionals, the authors studied the existence and stability of system (1), where the delays are constant. However, in this paper, for time-varying delays, by employing many different analysis techniques from [11], we establish the criteria ensuring the existence and exponential stability of a periodic solution of (1). Furthermore, if we take $r=2, m=1$, $q_{k}=1, \lambda_{i}=1, \alpha_{k i}=\beta_{k i}=\gamma_{k i}=\eta_{k i}=1 / 2$, then condition $\left(\mathrm{H}_{4}\right)$ is transformed into $\left(\mathrm{H}_{4}\right)^{\prime}$ as follows:

$$
\left(\mathrm{H}_{4}\right)^{\prime} \quad a_{i}^{-}-\sum_{i=1}^{n} b_{j i}^{+} M_{i}-\sum_{j=1}^{n} c_{j i}^{+} M_{i}-\sum_{j=1}^{n} \sum_{l=1}^{n}\left(d_{j i l}^{+}+d_{j l i}^{+}\right) N_{l} M_{i}-\sum_{j=1}^{n} \sum_{l=1}^{n}\left(e_{j i l}^{+}+e_{j l i}^{+}\right) N_{l} M_{i}>0,
$$


which is just the corresponding condition in [11]. One can derive the same results as [11]. That is, the criteria in [11] are the special case of Theorem 1 . Therefore, we extend and improve the earlier results in this sense.

If $d_{i j l}(t)=0$, then system (2) is transformed into the following model studied in [12].

$$
\begin{aligned}
\dot{x}_{i}(t)= & -a_{i}(t) x_{i}(t)+\sum_{j=1}^{n} b_{i j}(t) f_{j}\left(x_{j}(t)\right) \\
& +\sum_{j=1}^{n} c_{i j}(t) f_{j}\left(x_{j}\left(t-\tau_{i j}(t)\right)\right)+\sum_{j=1}^{n} \sum_{l=1}^{n} e_{i j l}(t) \\
& \times f_{j}\left(t-\sigma_{i j l}(t)\right) f_{l}\left(x_{l}\left(t-\sigma_{i j l}(t)\right)\right)+I_{i}(t), \quad t \neq t_{k}, \\
\Delta x_{i}\left(t_{k}\right)= & e_{i k}\left(x_{i}\left(t_{k}^{-}\right)\right), \quad t=t_{k} .
\end{aligned}
$$

Using a similar proof as above, one can obtain the main result of [12]. It is omitted here.

$$
\begin{aligned}
& \text { If } c_{i j}(t)=d_{i j l}(t)=e_{i j l}(t)=0 \text {, then }(2) \text { is transformed into the following model: } \\
& \dot{x}_{i}(t)=-a_{i}(t) x_{i}(t)+\sum_{j=1}^{n} b_{i j}(t) f_{j}\left(x_{j}(t)\right)+I_{i}(t), \quad t \neq t_{k} \\
& \Delta x_{i}\left(t_{k}\right)=e_{i k}\left(x_{i}\left(t_{k}^{-}\right)\right), \quad t=t_{k} .
\end{aligned}
$$

Similarly, we can obtain the sufficient conditions of the existence and exponential stability of a periodic solution to system (10), we omit it.

If one takes $r=2, m=2, q_{k}=1, \lambda_{i}=1, \alpha_{k i}=1 / 2$, then $\left(\mathrm{H}_{4}\right)$ is reduced to

$$
\left(\mathrm{H}_{4}\right)^{*} \quad a_{i}^{-}-\sum_{j=1}^{n} b_{j i}^{+} M_{i}>0 .
$$

The following corollary can be derived.

Corollary 2 Suppose that $\left(\mathrm{H}_{1}\right)-\left(\mathrm{H}_{3}\right),\left(\mathrm{H}_{4}\right)^{*},\left(\mathrm{H}_{5}\right)$ hold, then system $(10)$ has a unique exponentially stable $\omega$-periodic solution.

Remark 2 Corollary 2 is just the result of [27]. In [27], the impulses are required to be linear functions. However, without the above restrictions, we also establish the criteria ensuring the existence of an $\omega$-periodic solution which is exponentially stable. Thus we extend and generalize the earlier results.

In addition, if $d_{i j l}(t)=e_{i j l}(t)=0$, (2) reduces to the model studied in [8]. Similar results can be derived. In [8], the delay functions are required to be differential, but we do not need the restriction here.

On the other hand, in real world, by appropriately choosing parameters $r, m, q_{k}, \alpha_{k}, \beta_{k i}$, $\gamma_{k i}, \eta_{k i}$, one can see that many known assumptions can be included as special cases of $\left(\mathrm{H}_{4}\right)$, hence the discussion is interesting and valuable. 
a
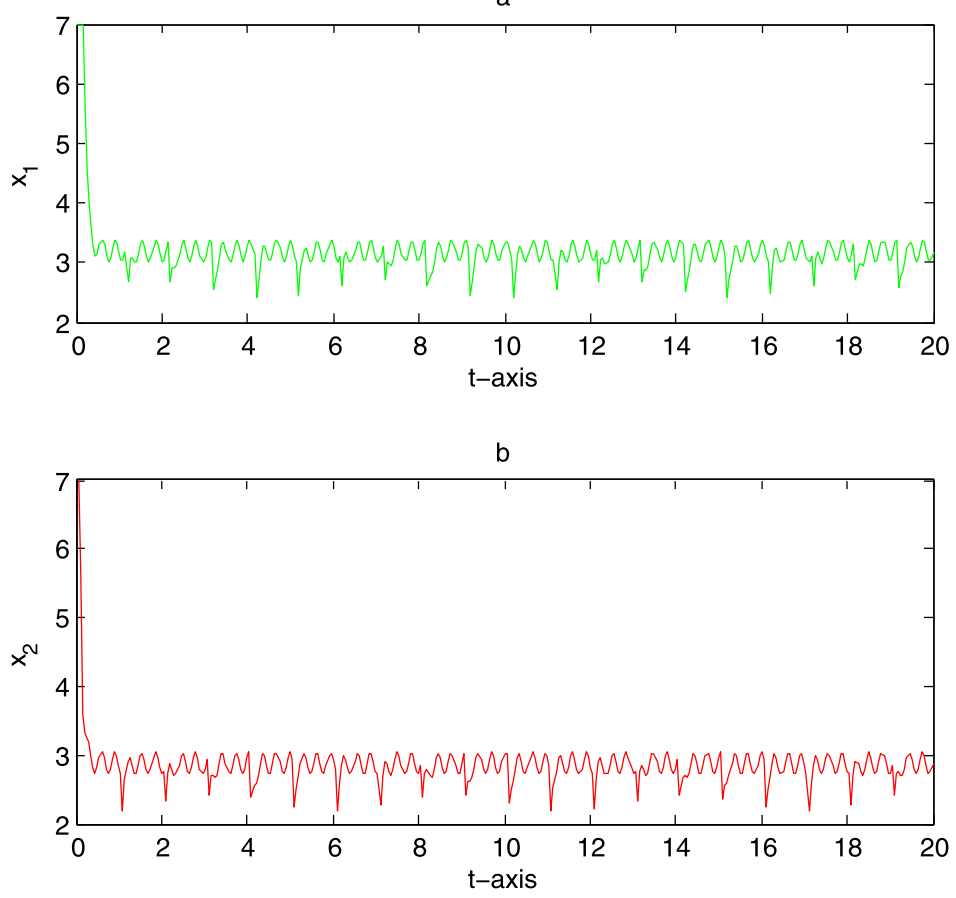

C

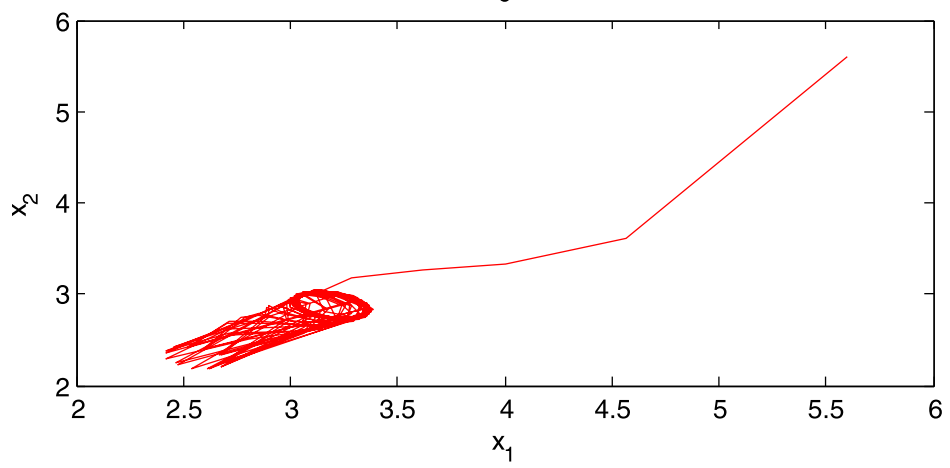

Figure 1 The dynamics of system (11). (a) Time series of $x_{1}(t)$, (b) time series of $x_{2}(t),(\mathbf{c})$ phase portrait of $x_{1}(t)$ and $x_{2}(t)$

\section{An illustrative example and simulations}

In this section, we give an example and simulations to show the validity of the main results.

Example Let

$$
\begin{aligned}
\dot{x}_{i}(t)= & -a_{i}(t) x_{i}(t)+\sum_{j=1}^{n} b_{i j}(t) f_{j}\left(x_{j}(t)\right)+\sum_{j=1}^{n} c_{i j}(t) f_{j}\left(x_{j}\left(t-\tau_{i j}(t)\right)\right) \\
& +\sum_{j=1}^{n} \sum_{l=1}^{n} d_{i j l}(t) f_{j}\left(x_{j}(t)\right) f_{l}\left(x_{l}(t)\right)+\sum_{j=1}^{n} \sum_{l=1}^{n} e_{i j l}(t) f_{j}\left(t-\sigma_{i j l}(t)\right) \\
& \times f_{l}\left(x_{l}\left(t-\sigma_{i j l}(t)\right)\right)+I_{i}(t), \quad t \neq t_{k}, \\
\Delta x_{i}\left(t_{k}\right)= & e_{i k}\left(x_{i}\left(t_{k}^{-}\right)\right), \quad t=t_{k},
\end{aligned}
$$


where

$$
\begin{aligned}
& n=2, \quad f_{j}(x)=\frac{|x+2|-|x-2|}{4}, \quad a_{1}(t)=10+\sin t, \\
& a_{2}(t)=12+\cos t, \quad \tau_{i j}(t)=\pi, \quad \sigma_{i j l}(t)=2 \pi, \quad e_{i k}\left(x_{i}\left(t_{k}^{-}\right)\right)=-0.4 x_{i}\left(t_{k}\right), \\
& B(t)=\left(b_{i j}(t)\right)_{2 \times 2}=\left(\begin{array}{cc}
\sin t & \cos t \\
\cos t / 4 & \sin t / 4
\end{array}\right), \quad C(t)=\left(c_{i j}(t)\right)_{2 \times 2}=\left(\begin{array}{cc}
2 \cos t & 2 \sin t \\
\sin t / 2 & \cos t / 2
\end{array}\right), \\
& d_{i j l}(t)=e_{i j l}(t)=4, \quad t_{k}=k \pi .
\end{aligned}
$$

Take $r=4, m=1, q_{k}=3, \alpha=\beta_{k i}=\gamma_{k i}=\delta_{k i}=1 / 3$. It is easy to verify that conditions of Theorem (1) hold. Therefore, by Theorem 1 , system (11) has a unique $\pi$-periodic solution, which is globally exponentially stable. By numerical analysis, the conclusion can be showed clearly, see Figure 1.

\section{Conclusion}

In this paper, by using the Halanay inequality and constructing Lyapunov functions, we address the existence and exponential stability of periodic solutions for a class of generalized cellular neural networks with impulses and time-varying delays. Easily verifiable sufficient conditions are obtained. The main results extend and improve some previously known results $[8,11,12,27]$. The criteria possess many adjustable parameters which provide flexibility for the design and analysis of a dynamical system. It is interesting and valuable.

\section{Competing interests}

The authors declare that they have no competing interests.

\section{Authors' contributions}

All authors read and approved the final manuscript.

\section{Author details}

${ }^{1}$ College of Science, Guilin University of Technology, Guilin Guangxi, 541004, P.R. China. ${ }^{2}$ Guizhou Key Laboratory of Economics System, Guizhou College of Finance and Economics, Guiyang Guizhou, 550004, P.R. China.

\section{Acknowledgements}

This paper is supported by National Natural Science Foundation of P.R. China (11161015, 11361012, 11161011), Natural Science Foundation of Guangxi (2013GXNSFAA019003, 2013GXNSFDA019001), and partially supported by National High Technology Research and Development Program 863 of P.R. China.

\section{Received: 10 August 2013 Accepted: 11 November 2013 Published: 03 Dec 2013}

\section{References}

1. Chua, LO, Yang, L: Cellular neural networks: theory and applications. IEEE Trans. Circuits Syst. 35, 1257-1290 (1988)

2. Venetianlr, PL, Roska, T: Image compression by cellular networks. IEEE Trans. Circuits Syst. I 45, 205-215 (1998)

3. Sun, CY, Feng, CB: Exponential periodicity and stability of delayed neural networks. Math. Comput. Simul. 66, 469-478 (2004)

4. Xu, BJ, Liu, XZ, Liao, XX: Global exponential stability of high order Hopfield type neural networks. Appl. Math. Comput. $174,98-116(2006)$

5. Xu, BJ, Liu, XZ, Liao, XX: Global asymptotic stability of high-order Hopfield type neural networks with time delays. Comput. Math. Appl. 45, 1729-1737 (2003)

6. Cao, JD: New results concerning exponential stability and periodic solutions of delayed cellular neural networks. Phys. Lett. A 307, 136-147 (2003)

7. Cao, JD, Wang, J: Absolute exponential stability of recurrent neural networks with Lipschitz-continuous activation functions and time delays. Neural Netw. 17, 379-390 (2004)

8. Jiang, HJ, Cao, JD: Global exponential stability of periodic neural networks with time-varying delays. Neurocomputing 70, 343-350 (2006)

9. Jiang, HJ, Teng, ZD: Global exponential stability of cellular neural networks with time-varying coefficient and delays. Neural Netw. 17, 1415-1425 (2004) 
10. Jiang, HJ, Li, ZM, Teng, ZD: Boundedness and stability for nonautonomous cellular neural networks with delay. Phys. Lett. A 306, 313-325 (2003)

11. Xiang, H, Yan, KM, Wang, BY: Existence and global exponential stability of periodic solution for delayed high-order Hopfield-type neural networks. Phys. Lett. A 352, 341-349 (2006)

12. Gu, HB, Jiang, HJ, Teng, ZD: Stability and periodicity in high-order neural networks with impulsive effects. Nonlinear Anal. (2007). doi:10.1016/j.na.2007.03.024

13. Wang, H, Liao, XF, Li, CD: Existence and exponential stability of periodic solution of BAM neural networks with impulse and time-varying delay. Chaos Solitons Fractals 33, 1028-1039 (2007)

14. Zhao, HY, Wang, GL: Delay-independent exponential stability of recurrent neural networks. Phys. Lett. A 333, 399-407 (2004)

15. Huang, LH, Huang, CX, Liu, BW: Dynamics of a class of cellular neural networks with time-varying delays. Phys. Lett. A 345, 330-344 (2005)

16. Zhou, J, Liu, Z, Chen, G: Dynamics of periodic delayed neural networks. Neural Netw. 17, 87-101 (2004)

17. Shao, YF: Exponential stability of periodic neural networks with impulsive effects and time-varying delays. Appl. Math. Comput. 217, 6893-6899 (2011)

18. Li, YK, Hua, YC, Fei, Y: Global exponential stability of delayed Cohen-Grossberg BAM neural networks with impulses on time scales. J. Inequal. Appl. (2009). doi:10.1155/2009/491268

19. Yang, XF, Liao, XF, Evans, DJ, Tang, YY: Existence and stability of periodic solution in impulsive Hopfield neural networks with finite distributed delays. Phys. Lett. A 343, 108-116 (2005)

20. Liao, XF, Liu, Q, Zhang, W: Delay-dependent asymptotic stability for neural networks with distributed delays Nonlinear Anal., Real World Appl. 7, 1178-1192 (2006)

21. Li, YK: Global exponential stability of BAM neural networks with delays and impulses. Chaos Solitons Fractals 24 279-285 (2005)

22. Li, YK, Xing, WY, Lu, LH: Existence and global exponential stability of periodic solution of a class of neural networks with impulses. Chaos Solitons Fractals 27, 437-445 (2006)

23. Li, YK, Liu, P: Existence and stability of positive periodic solution for BAM neural networks with delays. Math. Comput. Model. 40,757-770 (2004)

24. Yang, YQ, Cao, JD: Stability and periodicity in delayed cellular neural networks with impulsive effects. Nonlinear Anal., Real World Appl. 8, 362-374 (2007)

25. Gui, ZJ, Ge, WG: Periodic solutions of nonautonomous cellular neural networks with impulses. Chaos Solitons Fractals 32, 1760-1771 (2007)

26. Shao, YF, Dai, BX: The existence of exponential periodic attractor of impulsive BAM neural network with time-varying coefficients and distributed delays. Neurocomputing 73, 3123-3131 (2010)

27. Gui, ZJ, Ge, WG: Existence and uniqueness of periodic solutions of nonautonomous cellular neural networks with impulses. Phys. Lett. A 354, 84-94 (2006)

28. Beckenbach, EF, Bellman, R: Inequalities. Springer, Berlin (1965)

10.1186/1687-1847-2013-352

Cite this article as: Shao and Zhang: Stability and periodicity for impulsive neural networks with delays. Advances in Difference Equations 2013, 2013:352

\section{Submit your manuscript to a SpringerOpen ${ }^{\circ}$ journal and benefit from:}

- Convenient online submission

Rigorous peer review

- Immediate publication on acceptance

- Open access: articles freely available online

- High visibility within the field

- Retaining the copyright to your article 